

\section{Industrias creativas y tecnologías digitales en Colombia. Nuevas interacciones.}

\section{Felipe César Londoño}

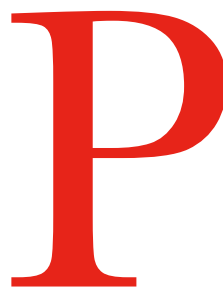
ocos intelectuales colombianos son capaces de hablar de manera tan incluyente de las transformaciones digitales que se han dado y se siguen dando en Colombia. Su basto conocimiento académico y sus desarrollos didácticos son el resultado de miradas adquiridas en las diferentes etapas que ha ido implementando en el mundo universitario y sus aplicaciones en los desempeños empresariales a los que ha contribuido con la puesta en marcha de plataformas para la consolidación de emprendimientos de gran calidad y competitividad profesional. Conocedor de la geografía colombiana ha contribuido a asimilar las relaciones entre las tradiciones, las deficiencias presupuestarias, las carencias formativas con la abundante creatividad, imaginación y fuerza de las regiones. Su artículo nos da buena muestra de todo ello.

\section{Presentación}

Las nuevas relaciones entre industrias creativas y tecnologías digitales generan una proliferación en la producción y el consumo de contenidos culturales en el mundo, gracias a las redes de distribución que multiplican los modelos de circulación existentes. En Colombia, el crecimiento exponen- cial de las industrias creativas va ligado, en gran parte, al impulso que las tecnologías digitales han tenido en los últimos años. Un ejemplo de ello se puede observar en el sector de la industria de animación digital, que creció en 800\% entre los años 2014 y 2016 (CASTELLANOS, 2017), lo que obliga a prestar especial atención a la manera como las empresas están enfrentado retos relacionados con la producción de contenidos, la calidad de las obras, los procesos complejos de posproducción, la visibilización y circulación de estas obras y su comercialización en los mercados nacionales e internacionales.

Efectivamente, las redes digitales y las tecnologías de información y comunicación han transformado los procesos de creación, producción, circulación, distribución de contenidos, rentabilización de los negocios creativos e intercambio de bienes y servicios culturales, y ello representa un desafío para el diseño de las políticas públicas del sector. Las tecnologías digitales hacen posible la divulgación masiva de los productos culturales y rompen los límites entre los creadores y los consumidores, instaurando con ello nuevos modelos de difusión y comercialización que obliga a estructurar nuevos modelos de propiedad intelectual y de derechos de autor tradicionales. 
Este artículo presenta acercamientos posibles entre las tecnologías digitales y las industrias culturales y creativas, a partir del análisis de los aspectos relacionados con la creación y la producción de obras y contenidos. Se profundiza en las transformaciones que se surten en las cadenas de valor de la industria creativa y se presenta, como ejemplo representativo, la plataforma digital del ClusterLab que se propone como dinamizadora de la industria de la animación digital en la región centro occidente de Colombia, en Panamá y Costa Rica, con base en el proyecto Fortalecimiento de la Cadena de Valor de la Industria de la Animación Digital que realiza la Comisión Económica Para América Latina y El Caribe (CEPAL) entre los años 2018 y 2019 .

\section{tivas}

Creación, tecnologías digitales e industrias crea-

Un campo de gran interés en el ámbito de las investigaciones sobre los impactos de las industrias culturales y creativas en la sociedad contemporánea es el de los procesos de adaptación de las tecnologías, tanto a las dinámicas de creación (generación de obras y contenidos) como a los de su circulación, visibilización, consumo y comercialización. Sin duda, la relación tecnología, medios de producción y creación ha estado presente desde el origen de la humanidad, pero es desde los inicios del siglo XX donde se populariza la utilización de los medios para la creación y divulgación de los contenidos culturales.

En general, se tiende a simplificar la relación creación-tecnologías al denominar una obra que se apropie de los recursos tecnológicos y utilice los medios de la industria del entretenimiento para difundir los contenidos. Lo complejo está en establecer la relación profunda entre las artes y las tecnologías disponibles, más allá de los soportes técnicos que las hacen posible. La cuestión más difícil, afirma Arlindo Machado, «es saber de qué manera pueden combinarse, contaminarse y distinguirse entre sí, instituciones tan distintas desde el punto de vista de sus respectivas historias, de sus temas o protagonistas y de la inserción social de cada una.» (2007).

Para Machado, si todo arte se hace con los medios de su tiempo, la creación que utiliza los medios digitales representa la expresión más avanzada de la creación artística y aquella que mejor expresa sensibilidades y saberes del ser humano de principios del tercer milenio. Y enuncia, para ello, tres relaciones posibles que la diferencian de las creaciones tradicionales:
- La manera como los creadores reinventan los medios, desviando la tecnología de su proyecto industrial para el que fueron concebidos.

- La forma como los contenidos culturales y artísticos develan los metalenguajes de la tecnología, realizando prácticas de alternativas críticas a los modelos actuales de normalización y control de la sociedad.

- El modo en que se reordena la industria del entretenimiento tradicional, a partir de productos artísticos en línea con nuevas sensibilidades emergentes.

La producción cultural en los ámbitos del arte se enfrenta hoy a indagar en las nuevas relaciones de las tecnologías y los procesos de creación, a profundizar en cuestiones que vinculan difusión, circulación y comercialización, y a ahondar en temas que tienen que ver con masificación; públicos y demandas en línea con los supuestos base de lo que hoy se conoce como las industrias creativas y culturales, un concepto apropiado por la institucionalidad política de los países relacionado, en gran parte, con presiones de orden económico nacional y mundial. Estos temas, que habían sido en parte analizados en el lúcido ensayo de Walter Benjamin sobre la fotografía y el cine, permanecen todavía vigentes. Benjamin enunciaba que, más allá de saber si todavía podemos considerar «artísticos» objetos y eventos propios de la industria del entretenimiento, lo que importa es percibir el surgimiento de nuevos fenómenos estéticos en la vida social a partir de esta industria (1989).

\section{Lo digital y las nuevas cadenas de valor en las in- dustrias creativas}

Si los asuntos relacionados con la creación de obras y contenidos se complejizan con las tecnologías digitales, también sucede lo mismo con los servicios cercanos a la actividad relacionada en la cultura. Labores tradicionales como la distribución, el transporte o la mensajería, que antes se consideraban informales en las cadenas de valor de la industria, hoy se definen desde un nuevo significado al convertirse en servidores de bases de datos, plataformas de circulación, minoristas en línea, entre otros, que son comercializados en el mercado digital. Diferentes autores afirman que estas actividades adquieren un nuevo valor, se consideran un activo para la economía digital y son un verdadero «contenido cultural» que tienen una demanda específica en el mercado (FEHÉR, K.; JUNAIDY, D. W.; LARASATI, D.; KOVÁCS, Á.; RAHARDJO, B., 2017).

Las industrias culturales y creativas caracterizan, de esta forma, un nuevo modelo de mercado basado en dinámi- 
cas organizacionales no estructuradas, donde todos los participantes de la cadena de producción en el mercado digital adquieren valor y todos ellos dependen de los contenidos que generan los creadores (UNESCO, 2015).

La digitalización ha transformado, por tanto, las tradicionales cadenas de valor que caracterizan al sector de la cultura y la economía. Mientras que algunos estudios afirman que la era digital genera un «alargamiento notable de la cadena de valor, con nuevas ventanas de comercialización y la presencia de nuevos intermediarios: ISP, portales y buscadores en Internet, gestores de multiplex en la radio y la televisión digitales, etc.» (ALBORNOZ, 2019), otros afirman que, en prácticas de conectividad, intercambio, colaboración e innovación, la cadena del proceso en las industrias culturales y creativas se vuelve mucho más corta y eficiente (FEHÉR, K.; JUNAIDY, D. W.; LARASATI, D.; KOVÁCS, Á.; RAHARDJO, B., 2017).

Por otra parte, el informe "Driving Innovation: Creative Industries Technology Strategy 2009-2012" describe la reconfiguración de la cadena de valor de las organizaciones culturales a partir de la digitalización y la forma como ella aporta de manera significativa, al incremento de ingresos y empleo en las industrias creativas, y a las formas de intermediación y los procesos de producción (UK TECHNOLOGY STRATEGY BOARD, 2009). En esta línea, el estudio sobre cultura como factor de innovación, dirigido por Pau Rausell Köster afirma que el contenido digital para las industrias creativas

ha promovido el desarrollo de nuevas aplicaciones y la integración y re-agregación de los recursos que intervienen en el proceso de producción", lo cual multiplica y diversifica los canales por lo que las obras culturales llegan a la demanda y que obliga a encontrar modelos de negocio adecuados para los productores de contenido para aprovechar el aumento del nivel de participación de los ciudadanos en las artes a través de medios digitales y electrónicos (2019).

Esta nueva relación entre economía y cultura es también analizada por Pier Luigi Sacco, miembro de la European Expert Network on Culture, quien establece una diferenciación entre los modelos históricos de esta relación (SACCO, 2011). Mientras que, en una primera fase de esta dependencia cultura y economía prevalecía el mecenazgo (un modelo de cultura 1.0, propio de una economía pre-industrial, que básicamente gira en torno al concepto de patrocinio), ahora, dice Sacco, prevalece la relación entre producción cultural y generación de valor económico (un modelo de cultura 2.0 que se caracteriza por la creciente expansión de las industrias creativas y los mercados culturales).

Cultura 3.0 implica ir más allá: su esencia está en que, gracias a las tecnologías, se multiplican los productores de contenidos, por lo que cada vez es más difícil distinguir entre creadores y usuarios culturales. Su sello distintivo es la transformación de las audiencias, es la constitución de comunidades de práctica que se expanden en las plataformas digitales. Más que los mercados culturales, la preocupación de estas industrias radica en el intercambio colaborativo y en la posibilidad de tener acceso libre a datos e información que posibiliten la generación de nuevas ideas, siempre vinculadas a las necesidades de un entorno cambiante.

\section{Las cadenas de valor en la Industria de la Anima- ción Digital: el proyecto CEPAL}

Una de las industrias creativas que más se ha consolidado en las últimas décadas en el mundo ha sido la de los contenidos digitales, una industria multimillonaria en donde se incluyen, entre muchos otros, la animación 2D y 3D, las series animadas, los videojuegos, las aplicaciones y los e-books. Un crecimiento rápido como consecuencia entre otros, del abaratamiento de los costos de adquisición y producción de tecnología digital: computadores, dispositivos móviles, consolas de videojuegos; además de la apropiación tecnológica por parte de las diversas comunidades. Los tradicionales modelos de negocio de la industria de los contenidos y el entretenimiento han cambiado radicalmente dando paso al surgimiento de otros más apropiados para la actualidad. La producción de contenidos como cine, animación y televisión ha migrado de lo analógico a lo digital dando paso a todo un ecosistema de empresas y producciones que van desde series animadas, VFX (visual effects), cine digital, videojuegos, apps, publicidad digital, contenido educativo, interactivo, libros digitales; para los cuales Internet ha sido un vehículo primordial. Es a través de las redes y las tecnologías digitales, que estos contenidos se difunden y se consumen logrando una descentralización de los usuarios o espectadores.

Se presencia de igual manera el surgimiento de una nueva generación de consumidores los cuales también crean contenido y lo comparten a través Internet. De esta forma, la publicidad y las estrategias de marketing tradicionales han perdido su eficacia ante espectadores activos que hoy se denominan interactores o prosumidores. Todos estos factores evidencian una industria y una cultura digital en continuo 
cambio siendo la inestabilidad una constante, donde las empresas que no se adaptan rápidamente a los cambios tienden a desaparecer.

Es en este contexto que se lleva a cabo el proyecto "Fortalecimiento de la cadena regional de valor de la Industria de la Animación Digital" en Costa Rica, Panamá y Colombia, que hace parte de un programa de fortalecimiento de una cadena regional de valor con alto potencial de encadenamientos para MIPYME en Mesoamérica (Proyecto EUROMIPYME CEPAL-UE). Las cadenas regionales de valor, para la GEPAL, constituyen un proceso de encadenamiento productivo en el que participan dos o más países con afinidad productiva, cercanía territorial y complementariedad comercial, con el objetivo de alcanzar una mayor articulación a partir de la integración de diferentes eslabones. De esta manera, el valor es «agregado» por diversos países cercanos con características económicas comunes o complementarias, a través de actividades que pueden describirse por un relativamente alto grado de dispersión. Para el caso de la industria de la animación digital en la región, el proyecto propone la realización de un diagnóstico, elaborado por consultores de cada uno de los tres países, que identifica detalladamente restricciones y oportunidades en el interior de cada eslabón de la cadena, así como sus vínculos reales y potenciales. El diagnóstico es discutido y validado en Mesas de Diálogo Regionales con la participación de los especialistas sectoriales, y a partir de allí se definen las líneas estratégicas que posibilitarán fortalecer la industria en la región.

\section{Incentivos para el crecimiento de la animación di- gital en Colombia}

El informe global de la industria de la animación, los efectos visuales y los juegos 2018-2020, de Research and Markets, evidencia que

el valor total de la industria de la animación global fue de US $\$ 254$ mil millones en 2017 y se proyecta que alcance los US\$270 mil millones para 2020” (s.p). La industria de la animación se ha ampliado de manera importante gracias al aumento de la transmisión por cable y televisión satelital, al acceso a internet de bajo costo, la utilización de dispositivos móviles y la trasmisión de video. Adicional a esto, están creciendo de manera vertiginosa las experiencias en realidad virtual y aumentada, acrecentando así la demanda de contenido de animación (2018).
Como lo describe Castellanos, para la animación digital se estimó una producción cercana a los US $\$ 198$ billones para el año 2010 en el mundo, con una tasa de crecimiento de $26 \% 2010$ y 2012 (Ca2017). Por otra parte, Berrendo y otros afirman que, en el período 2014-2016 en Iberoamérica, del total de los 2.000 estrenos en 20 países analizados, el $85,6 \%$ corresponden a estrenos de ficción y el $14,4 \%$ a obras de animación (BERRENDO y otros, 2017).

En particular en Colombia, y según el informe de Castellanos, el número de empresas de producción de contenidos en animación ha crecido un $800 \%$. Como políticas de apoyo a esta industria en Colombia, se han creado diversos incentivos a través de alianzas interinstitucionales, entre las que se destacan la Ley del Cine (Ley 814 de 2003) y la Ley 1556 del 2012 que reconocen el sector audiovisual como un motor de desarrollo productivo y económico del país; la convocatoria "Crea Digital", con categorías que integran la coproducción para el desarrollo de juegos de video, la coproducción para el desarrollo de contenidos transmediales y la coproducción para el desarrollo de series digitales animadas (convenio Ministerio de Cultura y el Ministerio de Tic); las convocatorias del Instituto Distrital de las Artes (Idartes); el Plan Vive Digital que viene ejecutándose desde finales de 2010 y, particularmente, a través de los ViveLabs en funcionamiento y encuentros como el Colombia 4.0, ha capacitado alrededor de mil quinientas personas en diseño e innovación de contenidos y aplicaciones digitales, modelos de negocio para aplicaciones móviles y desarrollo en Android, desarrollo web, animación 2D, videojuegos, programación y nuevas tecnologías; el programa Talento Digital del Ministerio TIC que busca formar talento humano en tecnologías de la información, las convocatorias del Fondo para el Desarrollo Cinematográfico para el desarrollo, producción y realización de largometrajes y cortometrajes animados; la Ley 1834 de 2017, por medio de la cual se fomenta la economía creativa Ley Naranja; y el Decreto 2120 del 15 de noviembre de 2018 por el cual se modifica la estructura del Ministerio de Cultura, entre otros.

En el ámbito específico de la ciudad de Manizales, la producción audiovisual y de contenidos digitales se ha dinamizado gracias a programas y proyectos que integran a las universidades e instituciones educativas en la ciudad (con programas de formación en pregrado, maestría y doctorado en campos del diseño, la animación y los contenidos digitales), a los laboratorios creados en los últimos años con el apoyo de los Ministerios de Cultura y de Tec- 


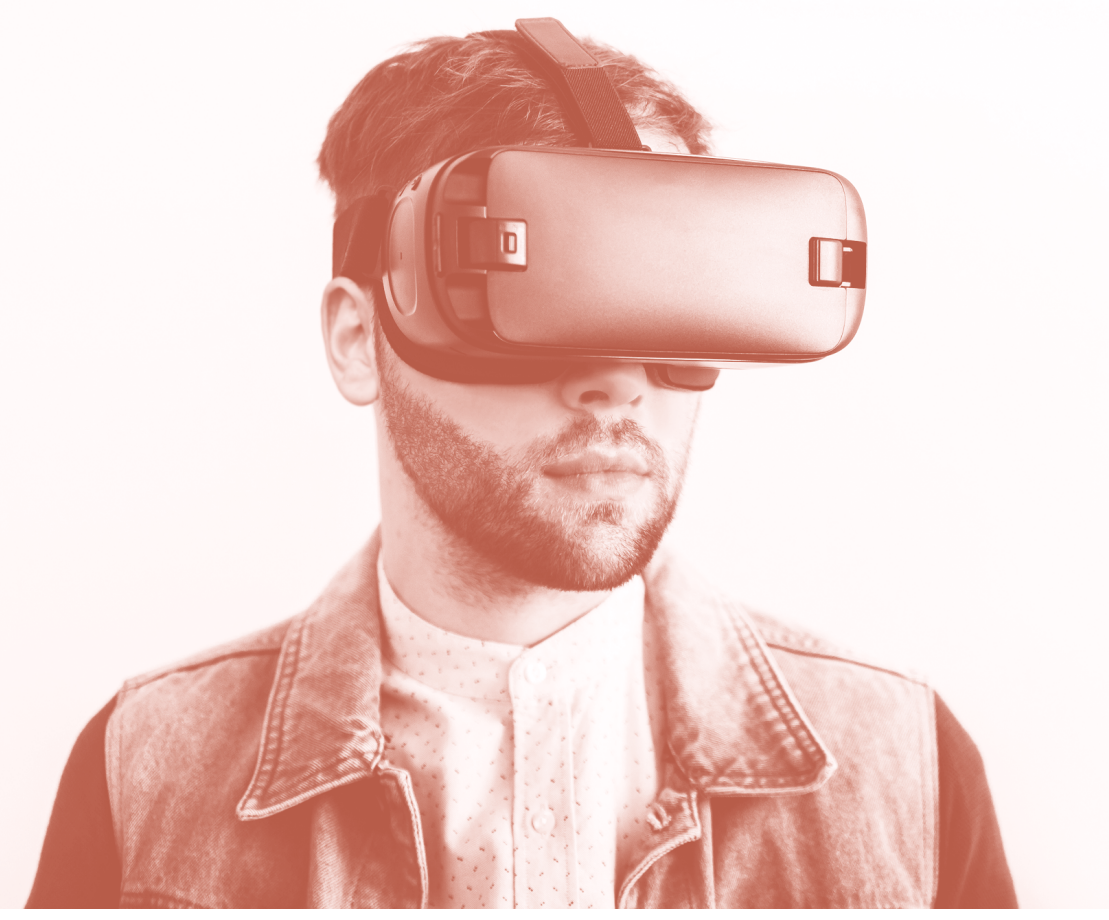

nologías de la Información y las Comunicaciones (como el MediaLab, LASO, el ViveLab Manizales y el Centro de Ciencia Francisco José de Caldas con sus laboratorios y espacios Maker); a eventos especializados en artes electrónicas y creación audiovisual (como el Festival Internacional de la Imagen, el Foro Académico Internacional en Diseño y Creación, el Mercado de Diseño, Arte Electrónico y Tecnología, el Festival Internacional de Teatro y el FICMA, Feria Internacional de Cine de Manizales, entre otros), y al trabajo en emprendimiento cultural por parte de varias organizaciones (Incubadora de Empresas Culturales, Nodo de Emprendimiento Cultural, Clúster de Industrias Creativas del Eje Cafetero, Neurocity de la Cámara de Comercio de Manizales, entre muchas otras). Todo lo anterior representa un avance significativo de relacionamiento para una ciudad considerada como campus universitario.

\section{Diagnóstico y conclusiones del estudio}

El diagnóstico para el análisis de la cadena regional de valor de la industria de la animación digital en el ámbito específico de Manizales se ejecutó con la participación de empresas registradas en la Cámara de Comercio de Manizales por Caldas y con instituciones de formación en ámbitos relacionados con el sector. Una vez identificados los participantes, el objetivo se centró en establecer una comunicación que permitiera conocer las dinámicas y procesos realizados al interior de cada empresa y/o institución de la industria en la ciudad de Manizales, ¿Cómo se estructuran? ¿Cuál es su eslabón clave? ¿Cómo se mueven en el mercado? ¿Cuáles son sus inversiones? Todo ello para conocer sus procesos a la hora de asumir un proyecto o servicio en calidad de coproducción, propio o para terceros. Los instrumentos de investigación en este proceso de interacción fueron La Co-Creación de la Matriz DOFA (Debilidades, 
Oportunidades, Fortalezas y Amenazas) para identificar los cuellos de botella por eslabones presentes en la cadena regional de valor; y la Entrevista Estructurada, que partió de la iniciativa de consolidar un diagnóstico sobre la industria de la animación digital en Manizales, con el objeto de conocer el estado actual y los cuellos de botella, de tal forma que se puedan tomar acciones para fortalecer los eslabones de dicha cadena.

Entre los hallazgos y restricciones más importantes que arroja el diagnóstico, se destacan:

- La escasa formación especializada en ámbitos específicos de la animación.

- Una insuficiente financiación para producir contenidos.

- Una competencia regional e internacional que impide una distribución adecuada.

- Poca visualización del modelo local y poco acceso a presupuestos para la circulación de las obras.

- Los imaginarios sociales sobre los contenidos de animación que, en muchas ocasiones, se vincula con las audiencias infantiles.

- Las múltiples dificultades en la venta de productos y en modelos de negocio y mercadeo efectivos.

Hallazgos similares se encontraron en las industrias de animación digital en Costa Rica y Panamá, y con ellos se establecen una serie de estrategias que permitan superar las restricciones para, con ello, fortalecer la cadena de valor de este sector de la economía en la región.

Para llevar a cabo el plan estratégico, se definió trabajar en la creación y adecuación de una plataforma digital común que permitiera la interacción de los actores de la industria de animación, de tal forma que se pueda promover:

- El desarrollo de proyectos conjuntos de animación digital entre Colombia, Panamá y Costa Rica, según capacidades regionales complementarias.

- El diseño de estrategias de marketing regional para promover el uso y consumo de los proyectos creados, entre comunidades, industrias, empresas, corporaciones, institutos de formación, entre otros.

- El desarrollo de un programa de acompañamiento empresarial para su fortalecimiento a través de la generación de modelos en gestión de negocios, donde los participantes puedan exponer sus planes, reciban feedback y asesoramiento, además de guías para hacer más eficaces sus proyectos mediante la construcción de pruebas piloto.

- El desarrollo de un repositorio digital con convocatorias para la posible financiación de los proyectos creados, ya sean de carácter público o privado, en ámbitos nacionales o internacionales.

- La creación de procesos de formación con base en las necesidades detectadas por la industria, y según los proyectos establecidos.

- La creación de un observatorio en animación digital que posibilite el análisis de tendencias, la competitividad, las necesidades que tiene el sector comercial, bolsas de trabajo, entre otros.

- La realización de eventos especializados en animación digital, para la visibilización de productos y obras de animación.

Para el desarrollo unificado de las estrategias que componen al Plan Estratégico, se propone la implementación de la plataforma ClusterLab (www.clusterlab.co), ya que promueve la interacción entre empresas y emprendedores culturales, mantiene un carácter colaborativo y apoya enfoques asocia-

En general, se tiende

a simplificar la relación creación-tecnología

al denominar una obra

que se apropie de los

recursos tecnológicos

y utilice los medios

de la industria del

entretenimiento para

difundir los contenidos. 
tivos para la gestión de los emprendimientos y las industrias creativas, a través de procesos de formación, actividades de investigación, vínculos con los actores del sector y otros clúster tecnológicos, lo cual permite la organización de ruedas de negocios e intercambios de conocimiento, como soporte a la incubación e internacionalización de las industrias creativas.

ClusterLab es una plataforma digital que promueve el Departamento de Diseño Visual de la Universidad de Caldas, en convenio con el Ministerio de Cultura, y que funciona a partir de las siguientes cuatro áreas estratégicas:

- OpenLab: Punto de encuentro y experimentación, que permite generar redes de creadores y productores interesados en desarrollar o colaborar en proyectos, prototipos, eventos o investigaciones enfocadas al fortalecimiento de la industria de la animación digital en la región.

- E-Learning: Promueve la formación virtual en ámbitos de la creación y la gestión en animación digital, a través de talleres, cursos, seminarios y conferencias con diferentes tecnologías como el streaming, el podcast, el repositorio digital de recursos educativos existentes en línea y la posibilidad de tener mentorías on-line especializadas.

- Networking: Para compartir y difundir portafolios de las industrias y generar comunidad entre empresas e industrias de animación, con nuevos enfoques asociativos.

- E-Commerce: Para ofrecer una guía sobre las formas de financiación, intercambio y comercio electrónico que pueden utilizan las empresas de animación digital que requieran acceder a apoyos económicos para llevar a cabo sus iniciativas.

En síntesis, el ClusterLab, como plataforma digital, crea un puente entre las industrias culturales y creativas y las tecnologías, a través de redes y alianzas con el sector empresarial, la academia y la oferta profesional. Esta, en el marco del proyecto de la CEPAL, se transforma en un espacio digital que determina escenarios de encuentro para que empresarios y emprendedores del sector de la animación digital de Costa Rica, Panamá y Colombia, desarrollen y promuevan sus iniciativas en un ambiente de formación, experimentación e intercambio abierto.

\section{Conclusiones}

El caso específico de la industria de la animación digital, a partir del proyecto de fortalecimiento de su cadena de valor liderado por la CEPAL, presenta las complejidades de una industria que se reinventa a partir de la evolución constante de sus eslabones, muchos de ellos afectados por los incrementos permanentes de las tecnologías.

Sin duda, las cadenas de valor de estas industrias se afectan con las dinámicas de la digitalización y ello obliga a tener en cuenta diversos escenarios tendenciales que es necesario analizar bajo la óptica de investigaciones que profundicen, no solo en las transformaciones de las cadenas de valor, sino también en las condiciones particulares de los artistas y creadores, que muchas veces no son tenidos en cuenta. Las plataformas digitales, como la del ClusterLab, evidencian la necesidad de promover la creación de espacios digitales abiertos que permitan las transferencias de conocimiento para resolver los problemas complejos en el ámbito de lo cultural y de la producción industrial.

Como afirma Albornoz,

en modo alguno el "cambio digital" supone una revolución, sino más bien se trata de una evolución, una línea de continuidad necesariamente contextualizada y determinada por las grandes transformaciones experimentadas en el último cuarto de siglo por los sectores dedicados a la producción informativa, comunicativa y cultural (2019).

La tecnología, en todo caso, sí posibilita una mayor experimentación, los intercambios de conocimiento, la disminución de costos de producción, la circulación en mercados internacionales, el trabajo colaborativo, la co-creación, las hibridaciones de sistemas tradicionales con los contemporáneos, la formación en línea, el fortalecimiento de las capacidades y del talento creativo, la búsqueda de contenidos inteligentes, la resistencia frente a los mercados globales, las redes de comunicación para búsqueda de fondos, entre otras.

Las industrias creativas deben repensarse, por tanto, a partir de estos nuevos modelos. Y para ello será necesario contemplar la importancia de lo local en perspectiva global, la integralidad de conocimientos y de contenidos, y las relaciones que se capitalizan en estas interacciones.

\section{Notas}

(1) Felipe César Londoño es un académico comprometido, líder de procesos transformadores y de programas relacionados en las nuevas dinámicas sociales que integran la ciencia, el arte y la tecnología. Profesor titular de la Uni- 
versidad de Caldas desde el año 1986, con doctorado en Ingeniería Multimedia de la UPC de Barcelona, ha participado en la creación de programas innovadores -como el Pregrado en Diseño Visual (1992), la Maestría en Diseño y Creación Interactiva (2007) y el Doctorado en Diseño y Creación (2010), reconocido por COLCIENCIAS como Doctorado de Excelencia en el 2013- con los cuales se ha fortalecido una comunidad académica transdisciplinar de gran impacto nacional, que por su alta calidad convoca a estudiantes, docentes e investigadores de diferentes disciplinas y universidades del país y del mundo. Ha sido rector de la Universidad de Caldas en Manizales y en la actualidad es decano Faculta de Artes y Diseño de la Universidad Jorge Tadeo Lozano en Bogotá.

\section{Bibliografía}

ALBORNOZ, L. Conclusiones de un estudio empírico: Las industrias culturales y las nuevas redes digitales, fecha de consulta: 15/05/2019, http://www.portalcomunicacion.com/dialeg/ paper/pdf/141_albornoz.pdf

BARANDIARAN, X. E.; ARAYA, D. Y VILA-VI-

NAS, D. (2015): "Ciencia: investigación participativa, colaborativa y abierta (v.1.0)" en Vila-Viñas, D. y Barandiaran, X. E. (eds.) Buen Conocer - FLOK Society. Modelos sostenibles y politicas públicas para una economía social del conocimiento común y abierto en el Ecuador, Quito, Ecuador, IAEN-CIESPAL.

BAUWENS, M. (2006): La economía politica de la Producción entre iguales, fecha de consulta: 02/05/2019, http://wiki. p2pfoundation.net/La_econom $\% \mathrm{C} 3 \% \mathrm{ADa}$ _pol $\% \mathrm{C} 3 \% \mathrm{AD}-$ tica_de_la_Producci $\% \mathrm{C} 3 \% \mathrm{~B} 3 \mathrm{n} \_$entre_iguales.

BENJAMIN, W. (1989): Discursos Interrumpidos I, Buenos Aires, Taurus.

BERRENDO PÉREZ, O. y otros (2017): Panorama Audiovisual Iberoamericano, Madrid, Departamento de Reparto y Documentación de EGEDA.

CASTELLANOS V., G. (2017): Propuestas de estímulo clúster de industrias creativas y de contenidos. Contrato 126 de 2017 / Convenio 106 de 2017, Bogotá, Cámara de Comercio de Bogotá, Secretaría Distrital de Cultura, Proimágenes Colombia.

CROSS, N. (2007): "From a Design Science to a Design Discipline: Understanding Designerly ways of Knowing and Thinking" en Michel, R. Design Research Now: Essays and Selected Projects. Basel, Birkhauser.

DANIEL, R. (2017): "The Creative Industries Concept: Stakeholder Reflections on Its Relevance and Potential in Australia”, Fournal of Australian Studies, no 41 (2), págs. 252-266.
FEHÉR, K.; JUNAIDY, D. W.; LARASATI, D.; KOVÁCS, Á. y RAHARDJO, B. (2017): Creative Industries and Digital Economy. Perspectives from Southeast Asia and Central Europe, fecha de consulta: 14/05/2019, https://www.researchgate. net/publication/319333661_Creative_Industries_and_Digital_Economy_-_Perspectives_from_Southeast_Asia_and_ Central_Europe

KÖSTER, P. y otros. La cultura como factor de innovación económica y social, fecha de consulta: 15/05/2019, http:// www.econcult.eu/es/publicaciones-sostenuto/

LONDOÑO, F. (2013): "Iniciativas creativas en las ciudades inteligentes: experiencias desde el Clúster Cultural del Eje Cafetero" en Ciudades Creativas. Espacio público y cultura en acción, Barcelona, Felix Manito, Fundación Kreanta. (2018): "Un Clúster para las Industrias Creativas, con énfasis en el sector de contenidos digitales" en INVENCOES, Brasil, Goiania, Editorial Universidad Federal de Goias.

LONDOÑO, F. y GÓMEZ, A. (2011): Paisajes y nuevos territorios (en Red). Cartografias e interacciones en entornos visuales y virtuales, Barcelona, Anthropos Editorial.

MACHADO, A. (2007): "Arte y medios: aproximaciones y diferencias" en Revista kepes, Colombia, Universidad de Caldas, vol. 3, n 2, págs. 145-163.

NICOLINI, G. (2016). "The Sharing Economy: What's Mine Is Yours and What's Yours Is Everyone's", Institute for the Integration of Latin America and the Caribbean, fecha de consulta: 02/05/2019, http://conexionintal.iadb. org/2016/09/05/economia-colaborativa-lo-mio-es-tuyo-y-lo-tuyo-es-de-todos/?lang=en

SACCO, P. (2011):. Culture 3.0: A new perspective for the EU 2014-2020 structural funds programming. OMC, Working Group on Cultural and Creative Industries, Europa, OMC.

UNESCO. Cultural Times. The first global map of cultural and creative industries, fecha de consulta: 01/09/2019, https:// unesdoc.unesco.org/ark:/48223/pf0000235710

UK Technology Strategy Board. (2009): Driving Innovation: Creative Industries Technology Strategy 2009-2012, Londres, UK Technology Strategy.

VV. AA. (2015): "Cultura: acceso y sostenibilidad en la era de la cultura libre (v.2.0). Documento de política pública 1.3" en Vila-Viñas, D. y Barandiaran, X. E. (eds.), Buen Conocer - FLOK Society. Modelos sostenibles y políticas públicas para una economía social del conocimiento común y abierto en el Ecuador. Quito, Ecuador, IAENCIESPAL.

WENGER, E. (2002): Comunidades de Práctica: aprendizaje, significado e identidad, España, Paidós. 


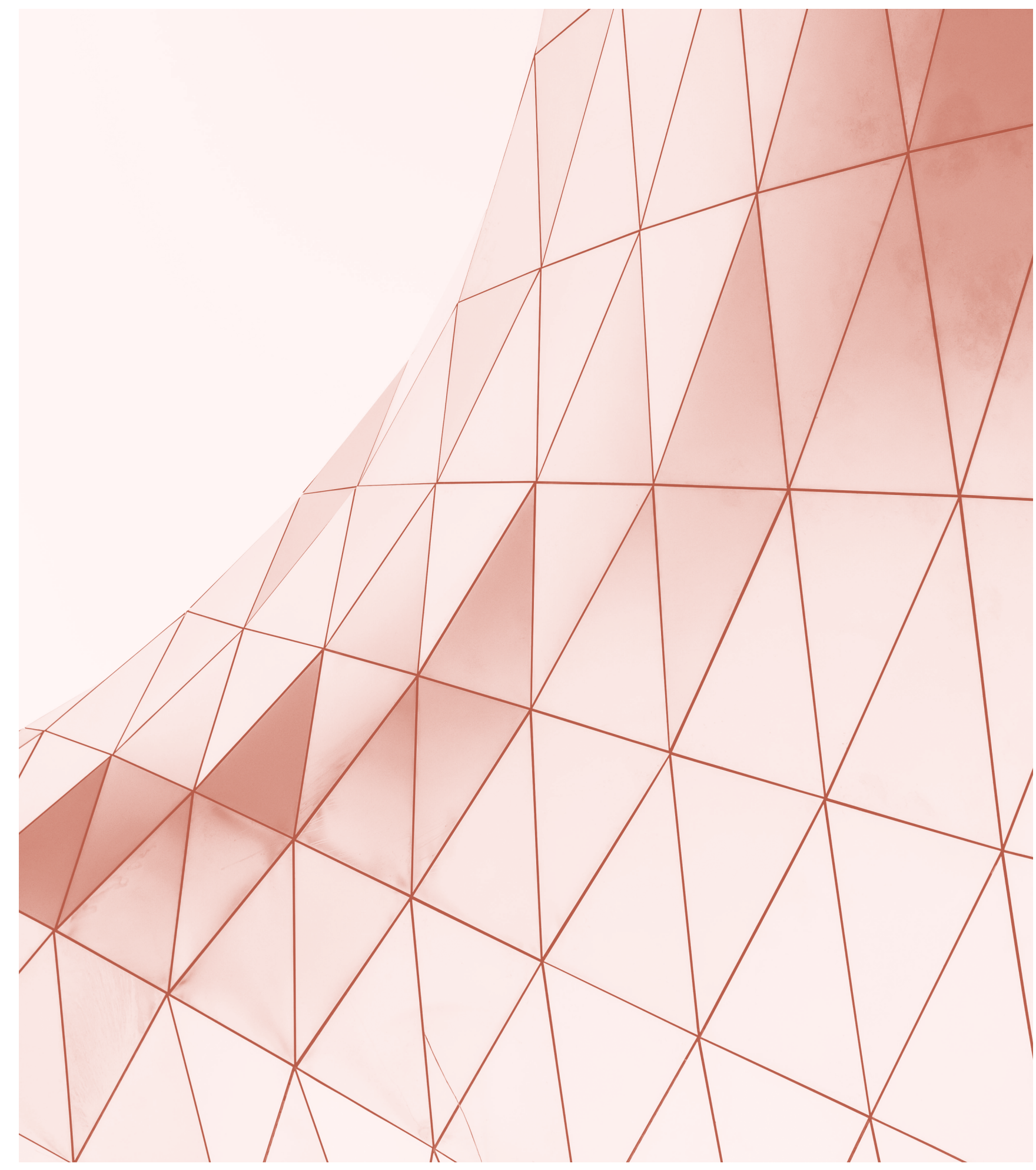

